\title{
Onlay ventral hernia repairs using porcine non-cross-linked dermal biologic mesh
}

\author{
E. T. Alicuben $\cdot$ S. R. DeMeester
}

Received: 5 July 2012/ Accepted: 28 January 2013/Published online: 12 February 2013

(C) The Author(s) 2013. This article is published with open access at Springerlink.com

\begin{abstract}
Introduction Ventral hernias are common and repair with mesh has been shown to reduce recurrence. However, synthetic mesh is associated with a risk of infection. Biologic mesh is an alternative that may be less susceptible to infection. Typically, the sublay position is preferred for mesh placement but this technique takes longer and has not been shown to have a lower recurrence rate than an onlay mesh. The aim of this study was to evaluate the outcome of complex ventral hernia repair using a porcine non-crosslinked biologic mesh onlay.

Methods A retrospective chart review was performed of all patients that had a ventral hernia repair with biologic mesh from January 2009 to March 2012. The operative procedure in all patients was an open repair with primary fascial closure (if possible) with or without external oblique component separation and porcine biologic mesh onlay.

Results There were 22 patients that had a ventral hernia repair, 19 primary and 3 recurrent. The majority were men, had hernia grade 3 or 4 , and developed the hernia after an esophagectomy or gastrectomy for cancer. All but one had primary closure with a porcine biologic mesh onlay. One patient was bridged for loss of domain. A bilateral external oblique component separation was added in 16 patients (73\%). The median hospital stay was 7 days. There were two superficial wound infections, one with exposed mesh, but no patient required mesh removal. A seroma requiring intervention developed in 6 patients $(27 \%)$ and resolved
\end{abstract}

E. T. Alicuben · S. R. DeMeester $(\square)$

Department of Surgery, Keck School of Medicine,

The University of Southern California, 1510 San Pablo St,

Suite 514, Los Angeles, CA 90033, USA

e-mail: sdemeester@surgery.usc.edu with pig-tail drainage. At a median follow-up of 7 months, there has been no hernia recurrence apart from the patient that was bridged.

Conclusions Porcine non-cross-linked biologic mesh overlay has excellent short-term results in patients at increased risk for mesh infection. No patient required mesh removal, and there have been no recurrent hernias in patients with primary fascial closure. Biologic bridging is not effective for long-term abdominal wall reconstruction.

Keywords Ventral hernia - Component separation . Biologic mesh · Onlay ventral hernia repair .

Open ventral hernia repair - Non-cross-linked porcine dermal biologic mesh

\section{Introduction}

There are 4-5 million laparotomies performed in the United States annually, and an incisional hernia will develop in $10-15 \%$ of these patients [1]. Ventral hernia repair is a common procedure, with some 250,000 procedures performed annually, but recurrence rates are high and increase with each subsequent attempt at repair [2,3]. The use of synthetic mesh has been shown in a randomized prospective trial to reduce recurrence after ventral hernia repair [4]. However, synthetic mesh is associated with a number of potential complications including erosion, fistula formation, and infection. One of the leading causes of a recurrent hernia is infection, and the risk of infection is increased in patients with a history of prior wound infection, emphysema, diabetes, obesity and in those taking steroids or smoking cigarettes [5]. These comorbid conditions are common in patients with a ventral hernia and may be a reason to consider use of a non-synthetic mesh. 
An alternative to synthetic material is a biologic mesh. One potential advantage of biologic mesh is that it may be less susceptible to contamination and infection. However, there is little data on the efficacy of biologic mesh for ventral hernia repair. Our ventral hernia patient population consists primarily of patients that had a laparotomy for esophageal or gastric cancer. Most were in follow-up for their cancer, and at risk for recurrent disease at the time the ventral hernia was diagnosed. Since recurrence is generally treated with chemotherapy, an indolent or active mesh infection related to ventral hernia repair would compromise the treatment options. The aim of this study was to review our results for repair of complicated ventral hernias with a porcine non-cross-linked biologic mesh onlay.

\section{Methods}

A retrospective chart review was performed of all patients who underwent ventral hernia repair with use of a biologic mesh from January 2009 to March 2012 by a single surgeon. The risk of infection was assessed using the hernia grade system as described by the ventral hernia working group [5]. In addition, at the time of the ventral hernia repair, wounds were classified into one of the three categories: clean, clean-contaminated (exposure to gastrointestinal tract without spillage or necrosis), or contaminated (hollow-viscous necrosis or gastrointestinal tract spillage). Postoperatively, patients were seen at routine intervals, and an abdominal examination was performed to evaluate the integrity of the repair. Patients undergoing cancer followup had abdominal CT scans every 3-6 months and these were reviewed for both cancer recurrence and the status of the ventral hernia repair. This study was approved by the IRB of the University of Southern California.

The operative procedure in all patients consisted of a midline laparotomy through the old incision with mobilization of the adhesions to the undersurface of the fascia circumferentially. Old mesh, if present, was removed. Old jejunostomy tube sites were selectively taken down to facilitate trans-fascial suture placement. One patient had a colo-cutaneous fistula, and a concomitant colon resection was performed with takedown of the fistula. In all patients, subcutaneous flaps were raised bilaterally until the muscle fibers of the external oblique were visible laterally. The fascial edges were trimmed to healthy tissue and the hernia sac excised. The fascial edges were approximated in the midline and tension assessed. If any significant tension was present, a bilateral external oblique component separation was performed as described by Ramirez et al. [6]. Transfascial 0 proline sutures were placed lateral to the site of the component separation and 3 knots were placed to prevent any bowel from becoming trapped in the loop of the suture. After circumferential trans-fascial suture placement, the midline was closed with figure-of-eight 0 proline suture. The ends of the midline closure sutures were not cut and the needles left attached. Once the midline was closed, an appropriately sized non-cross-linked porcine dermal biologic mesh was selected (XenMatrix ${ }^{\circledR}$ in all but 1 patient), trimmed to fit, and placed as an onlay. The inferior and left lateral trans-fascial sutures were placed through the mesh using the native swedged on needle and a free needle for the other end of the suture to create a mattress stitch through the mesh. After several sutures were placed on the left side of the mesh, it was pulled firmly toward the right side, and the right lateral trans-fascial sutures were placed in similar fashion. Before going back to place more left lateral trans-fascial sutures, the midline closure suture ends were brought through the mid-portion of the mesh, similar to how the trans-fascial sutures were placed, and tied to secure the middle of the mesh to the fascia. This sequence continued until all the trans-fascial sutures and all the midline sutures were brought through the mesh and tied (Fig. 1). The goal was to place as much tension as possible on the mesh and relieve tension on the midline closure. Two closed-suction Jackson-Pratt drains were placed, one under each flap, and the deep

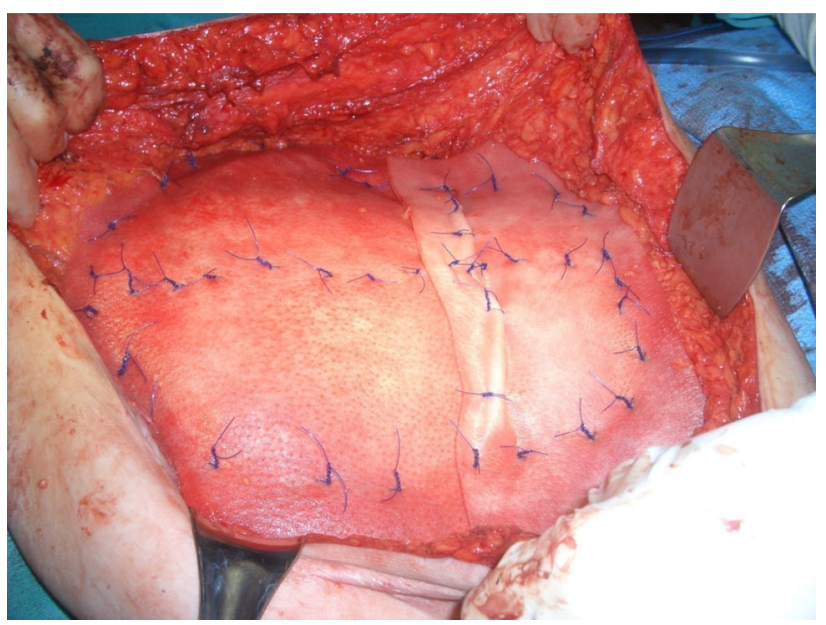

Fig. 1 Completed repair of large ventral hernia. The fascia has been closed in the midline after bilateral external oblique component separations. A $19 \times 29$ sheet of XenMatrix has been secured in place with \#1 proline mattress sutures placed trans-fascially through the mesh circumferentially. Note the trans-fascial sutures are placed lateral to the site of the component separation and the mesh covers this area bilaterally. The midline figure-of-eight proline sutures have also been brought through the mesh to anchor the mesh to the fascia in the midline. The defect was so large that 2 pieces of mesh had to be used and were sewn together. Note the tautness of the mesh. Tension is deliberately placed on the mesh to minimize tension on the midline fascial closure during early healing. The space will be drained with two \#10 Jackson-Pratt drains, the deep subcutaneous tissues sewn together and attached to the mesh with $2-0$ vicryl, and the skin closed with staples to complete the procedure 
subcutaneous tissues were sutured together and to the mesh with 2-0 vicryl. The umbilicus was tacked down to the mesh with 2-0 vicryl sutures since in all cases it was mobilized off the fascia to facilitate mesh placement circumferentially over the primary midline closure. A binder was applied in the operating room, and an epidural catheter was used for pain control. Patients were typically discharged with the JP drains in place with instructions to record the outputs. Drains were removed in the clinic when the output dropped to under $15 \mathrm{cc}$ per day. Patients were restricted to lifting no more than 5 pounds for 3 months after the repair.

\section{Statistics}

Values are presented as median and interquartile range unless otherwise specified.

\section{Results}

During the three years of the study, 22 patients had repair of a complex ventral hernia that included use of a noncross-linked porcine biologic mesh. Characteristics of the patients are shown in Table 1. The majority of the patients were men, had hernia grade 3 or 4, and developed the ventral hernia after an esophagectomy or gastrectomy for cancer. The non-cross-linked porcine biologic mesh was placed as an onlay after primary midline fascial closure in

Table 1 Patient characteristics

\begin{tabular}{ll}
\hline & $n=22$ \\
\hline Male:female & $15: 7$ \\
Age & $66(59-68)^{\mathrm{a}}$ \\
BMI & $26(24-35)^{\mathrm{a}}$ \\
Cancer operation led to hernia yes:no & $15: 6$ \\
Received neoadjuvant therapy & 7 \\
Hernia grade & \\
Grade 1 & 2 \\
Grade 2 & 3 \\
Grade 3 & 16 \\
Grade 4 & 1 \\
Hernia type & \\
Primary & 19 \\
Recurrent & 3 \\
Wound classification & \\
Clean & 15 \\
Clean-contaminated & 6 \\
Contaminated & 1 \\
\hline
\end{tabular}

${ }^{\mathrm{a}} \mathrm{IQR}$
Table 2 Postoperative morbidity

\begin{tabular}{ll}
\hline & $n=21$ \\
\hline Seroma & 6 \\
Wound infection & 2 \\
Recurrence & 1 (after bridge repair) \\
\hline
\end{tabular}

21 patients and as a bridge in 1 patient with loss of domain. A concomitant bilateral external oblique component separation was performed in 16 patients $(73 \%)$. There was one perioperative death at 5 days from a myocardial infarction. Complications are shown in Table 2. The median hospital stay was 7 days and $86 \%$ of patients left the hospital with one or more closed-suction drains in place. Two patients, both with multiple medical comorbidities, developed a wound infection, one superficial and one with exposure of the mesh. Both were treated successfully with a wound vac. A seroma was seen by CT scan in 9 patients (42\%), but required drainage in only 6 patients $(27 \%)$. All seromas were treated successfully with a pig-tail drain placed by ultrasound or CT guidance (single pig-tail in 5 patients and 2 in one patient) (Fig. 2a, b). The median time until all drains were removed was 30 days (range 6-211 days). One patient, on steroids for lung disease, developed a Spigelian hernia 9 months postoperatively at the site of a trans-fascial suture that had pulled through the abdominal wall (Fig. 3a, b). This was repaired laparoscopically.

The median follow-up was 7 months (IQR 2-14 months) in all patients and exceeded 1 year in 7 patients. Follow-up included at least one abdominal CT scan in 15 patients (71\%). Serial CT scans out to 18 months in one patient showed some residual mesh in place. The only hernia recurrence was in the patient that was bridged for loss of domain. His operation was to be staged with a planned reoperation and primary fascial closure after 3 months, but the patient did not re-present until over a year later at which time a recurrent hernia was identified on abdominal CT scan. At reoperation 19 months after implantation, the biologic mesh was still partially present and was composed of 2 layers (Fig. 4a, b). The outer layer was firmly incorporated into the subcutaneous tissue, while the inner layer was adhered to the omentum and colon. The patient was bridged again, this time with a synthetic mesh, for continued loss of domain. Another patient underwent re-exploration for cancer recurrence 4 months after abdominal wall reconstruction with a XenMatrix ${ }^{\circledR}$ onlay. There was complete incorporation of the subcutaneous flaps with the mesh, and histology showed extensive neovascularity and fibroblast infiltration into the mesh (Fig. 5a-c). 
A

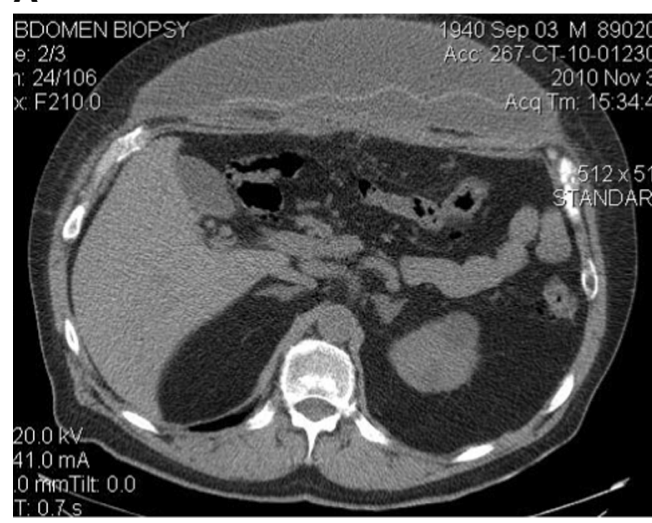

B

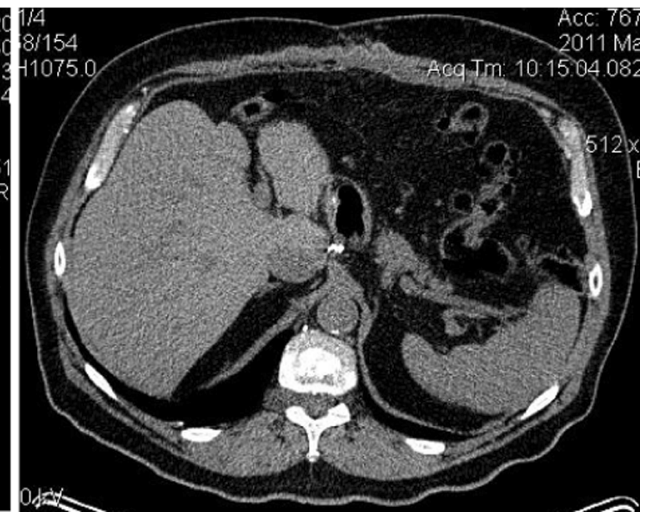

Fig. 2 Slices from an abdominal CT scan showing a large seroma above mesh with portion of mesh in fluid, and $\mathbf{b}$ resolution of seroma and intact hernia repair after pig-tail drainage. The mesh is still visible above the fascial closure

A

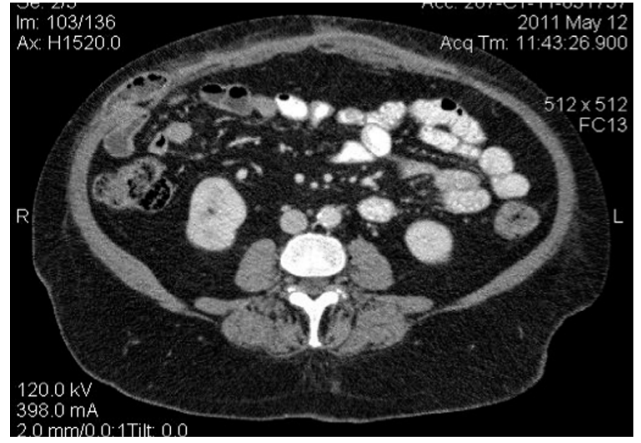

Fig. 3 a Abdominal CT scan and b laparoscopic picture of Spigelian hernia in site where a trans-fascial suture had pulled through the abdominal wall in a patient on large doses of steroids for lung disease

\section{A}

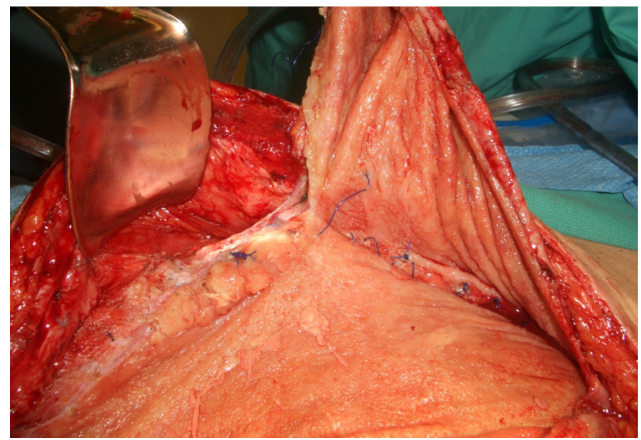

Fig. 4 Reoperation 19 months after bridging a patient with loss of domain with XenMatrix. a The mesh has developed into two layers with the subcutaneous tissues incorporated into the superficial part of the mesh. The deeper portion of the mesh is against the viscera. The site of the hernia is just below the retractor where the mesh has separated from the fascia. Note the absence of any form of

\section{Discussion}

Mesh has been used to reinforce repair of a ventral hernia since the early 1900s. The initial materials included silver,
B

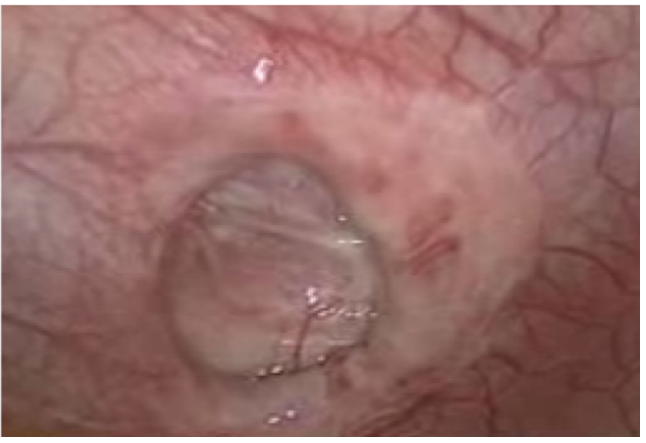

that had ventral hernia repair. Note the mesh is still visible on the CT scan after almost a year, and the ventral hernia repair is intact

B

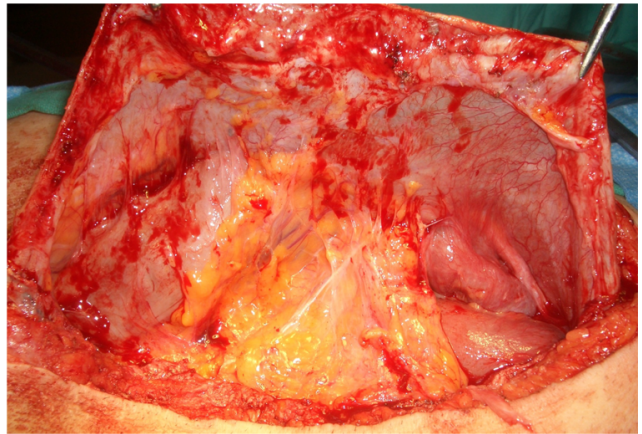

reconstructed abdominal wall. b The deeper portion of mesh has been separated laterally from the fascia and is being held up by clamps. Neovascularity and adhesions of the omentum and colon to the mesh are visible. The mesh with attached viscera was tucked into the abdomen with no effort to remove it, and since the patient continued to have loss of domain he was bridged with synthetic mesh

tantalum gauze, and stainless steel [7]. However, the introduction of Marlex in 1959 revolutionized hernia repair and was the foundation for a variety of subsequent synthetic mesh materials and designs. While the use of 

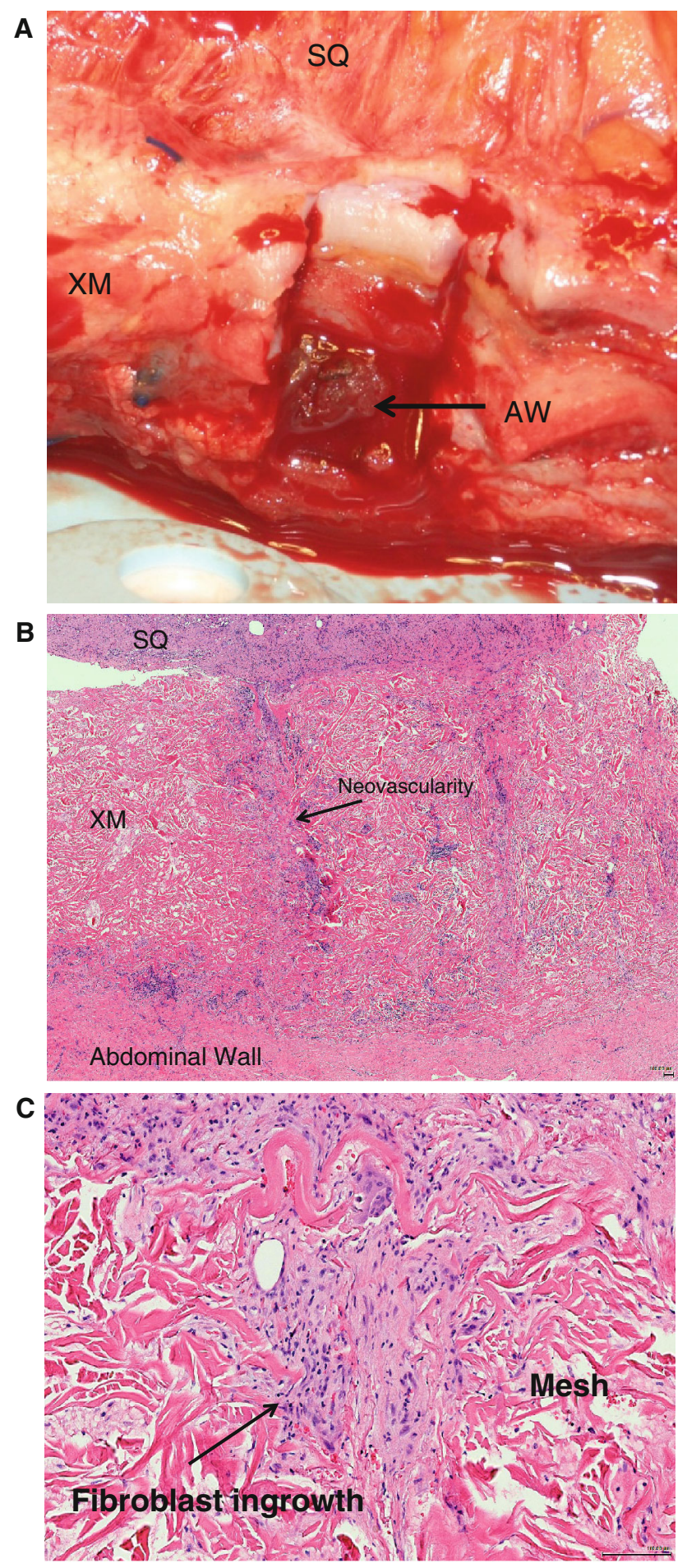

Fig. 5 Re-exploration at 4 months in a patient with cancer. a A fullthickness resection of subcutaneous tissue, XenMatrix mesh, and native abdominal wall (at site of arrow) for histology. Note the incorporation of the subcutaneous tissue (SQ) into the mesh (XM), and the mesh to the abdominal wall (AW). b Histology showing neovascularity going entirely through the mesh from the abdominal wall to the subcutaneous tissue (arrow). c Higher magnification showing infiltration of fibroblasts into the open collagen structure of the mesh $(\mathrm{XM})$ synthetic mesh has been proven to reduce recurrence, it is important to recognize that although synthetic mesh is permanent, the use of synthetic mesh does not mean permanent repair of a ventral hernia. Long-term follow-up of a randomized controlled trial showed that by 10 years, $32 \%$ of patients that had a ventral hernia repair with proline or Marlex mesh had developed a recurrent hernia [8]. Further, the use of synthetic mesh is associated with complications including shrinkage of the mesh with subsequent chronic pain and/or hernia recurrence, erosion, and fistula formation. When placed intra-abdominally, synthetic mesh can lead to severe adhesions to the viscera, making reoperation difficult and increasing the risk for enterotomies. Synthetic mesh is also associated with a risk for both early and delayed infection, and once infected, synthetic mesh often needs to be removed. Infected mesh is also a leading cause of hernia recurrence [9, 10]. In a recent review of 33,832 procedures, Choi et al. [2] reported that the use of mesh in clean-contaminated cases was associated with a significantly increased risk of superficial, deep and organ/space infections, wound disruption, pneumonia, and sepsis compared to non-use of mesh. Based on these findings, the authors recommended that synthetic mesh should not be used in clean-contaminated or contaminated ventral hernia repairs.

In contrast to synthetic mesh, biologic mesh is not associated with a significant risk of erosion or fistula formation and is likely less susceptible to contamination. In a rat study, Harth et al. [11] showed that while inoculation of synthetic mesh with Staphylococcus aureus was never cleared, the majority of inoculated biologic meshes were culture negative when explanted, particularly non-cross-linked porcine dermal grafts. As a consequence of this benefit, the ventral hernia working group [5] recommended that a biologic mesh be considered for Hernia Grades 2 and higher. In a recent review of mesh types, Shankaran et al. [7] listed the characteristics of an ideal mesh and noted that the only mesh type to fulfill all of the criteria was biologic mesh. The concept of a biologic mesh is that it is an acellular collagen matrix that allows neovascularity and ingrowth by native fibroblasts, with gradual incorporation and replacement of the mesh by host tissue. Current biologic meshes come from human dermis and several animal types and tissues. The biologic mesh can be cross-linked or non-cross-linked, but non-cross-linked mesh incorporates more reliably and is currently preferred in many centers [12].

The biologic mesh types that have been reported for ventral hernia repair include porcine submucosa, human dermis, and porcine dermis. The use of porcine submucosa (Surgisis; Cook Surgical, Bloomington, IN) for ventral hernia repair was associated with a $41 \%$ complication rate in a study by Helton et al. [13], and the authors advised 
caution with this mesh, particularly in critically ill patients and those with dirty wounds. In another study, Surgisis was compared to Alloderm (LifeCell Corp, Branchburg, NJ) for ventral hernia repair, and the authors reported a high incidence of seroma formation and abdominal discomfort related to poor tissue integration with Surgisis, and diastasis and hernia recurrence with Alloderm related to stretching of the graft [14]. Other studies have confirmed that since Alloderm increases in length by $30-50 \%$ when placed under tension due to the elastin fibers in the graft, there is a high incidence of laxity in the abdominal wall and hernia recurrence, particularly when it is used to bridge a fascial defect $[15,16]$. In a direct comparison of Alloderm versus soft polypropylene mesh as an underlay in clean cases, Ko et al. [17] reported a significantly higher recurrence rate with Alloderm (61\%) compared to the synthetic mesh (12\%). Finally, in a review of published literature on biologic mesh use for hernia repair, Hiles et al. [18] concluded that Alloderm was associated with the highest failure rate with the shortest average follow-up of all the materials.

Given the poor results with Surgisis and Alloderm, interest has shifted to the use of non-cross-linked porcine dermis as a biologic mesh for ventral hernia repair. The two available grafts are XenMatrix (Davol, Warwick RI) and Strattice (LifeCell Corp, Branchburg, NJ). Pomahac et al. [19] reported on the use of XenMatrix as a subfascial underlay in 16 patients. Seromas developed in $21 \%$ and there was one recurrent hernia $(7 \%)$ at a mean follow-up of 16.5 months. In an expansion of that series, the authors recently reported on 40 patients, 32 with primary fascial closure, at a mean follow-up of 40.1 months [20]. A seroma developed in $21 \%$ of patients, and the XenMatrix was removed in 1 patient for infection when it was used to reconstruct the abdominal wall after removal of infected synthetic mesh. The hernia recurrence rate remained at 7.6 \%. In another study, Byrnes et al. [21] used XenMatrix as an underlay $(84 \%)$ or bridge $(16 \%)$ repair in 57 patients. They identified 4 recurrences $(7.2 \%)$ at a mean follow-up of 30.6 months, but noted that all recurrences were within 3 weeks of the repair and all were in patients that were bridged. There have been three reports on the use of Strattice for ventral hernia repair and one case series of three patients [22-25]. Rosen et al. [22] reported on a small group of 12 patients with combined ventral and parastomal hernias repaired with a retrorectus technique using Strattice mesh. Postoperative complications occurred in $33 \%$ of patients, and at a mean follow-up of 14 months a hernia recurred in two patients. In a larger series of 80 patients with infected or contaminated ventral hernias, Rosen et al. [23] noted a $30 \%$ recurrence rate with Strattice mesh at one year with intraperitoneal repair and a $20 \%$ recurrence when the mesh was used as a sublay after primary closure and component separation. Lastly, Patel et al. [24] reported on 41 patients that had primary fascial closure with Strattice underlay and component separation in nearly everyone. Complications occurred in $24 \%$ of patients and were largely related to skin necrosis. At a mean follow-up of 1.3 years they reported no recurrent hernias.

In this series, we used XenMatrix as an onlay for reinforcement of ventral hernia repair. We deliberately placed as much tension as possible on the mesh to minimize tension on the primary midline fascial closure and made liberal use of an external oblique component separation to further reduce tension. A seroma requiring intervention developed in $27 \%$ of patients, but all were successfully treated by pigtail catheter drainage. No patient had flap necrosis. No mesh was removed for infection despite the fact that $77 \%$ of patients were classified as having hernia grades 3 or 4 , and the wound was clean-contaminated or contaminated in $32 \%$ of patients. Based on the recommendations of Choi et al. [2], synthetic mesh use would have been contraindicated in these patients given the significantly increased risk of infection. The only hernia recurrence was in a patient that was bridged as a planned staged procedure for loss of domain, but the patient failed to present for the second stage of the repair until 19 months later. None of the patients that had primary fascial closure and XenMatrix onlay have developed a recurrent hernia, including 7 patients with a minimum of 12 months of follow-up.

We recognize that this is a small series with short overall follow-up. However, there are several factors that make this study unique. For one, this is the first study to report the use of XenMatrix or any non-cross-linked porcine dermal graft as an onlay for ventral hernia repair. Most centers consider the Rives-Stoppa repair with sublay of the mesh below the rectus to be the gold standard for ventral hernia repair. Part of the rationale for this approach is to keep the mesh as deep as possible to avoid contamination should there be a superficial wound infection. However, the use of a biologic mesh changes this concern since exposure of a biologic mesh to a superficial wound infection does not have the same implications as a synthetic mesh. In our series, there were two patients with superficial wound infections, one with exposed mesh, and both healed with wound vac treatment. No mesh was removed for infection. From the standpoint of hernia recurrence, there is little data to suggest that sublay is superior to an onlay. In 2011, a Cochrane review was published comparing onlay and sublay mesh for ventral hernia repair. The operative times were shorter with a synthetic mesh onlay but hospital stay, surgical pain, overall complication rate, and hernia recurrence rate were similar for the onlay and sublay technique [26]. In our experience, the onlay technique allowed for the prompt addition of an external oblique component separation if deemed necessary since bilateral subcutaneous 
flaps had already been raised. In our opinion, the substantial increase in abdominal wall movement that accompanies this release makes it an important addition in any patient with even mild tension on the midline closure.

The most common morbidity we encountered was seroma formation, likely as a host response to the biologic mesh since our incidence was higher than reports in the literature for synthetic mesh onlay $(23 \%)$ and in comparison with our own historical data (7.1\%, unpublished data) [27]. This experience has led us to consider the use of aerosolized talc in the space beneath the subcutaneous flaps given recent evidence showing a significant reduction in seroma formation, length of indwelling drains, and cellulitis with the use of talc [28]. However, it is important to realize that a seroma in a patient with a biologic mesh is completely different than a seroma in a patient with a synthetic mesh. The same level of concern regarding introducing infection with drainage of a seroma is not there with a biologic mesh compared to a synthetic mesh, and some of the pig-tail catheters we placed to drain a seroma stayed for several weeks with no infectious complications. Therefore, while a seroma was common in our series, pigtail catheter drainage was easy, successfully resolved the seroma in all patients, and was not associated with any complications.

Another unique aspect of our study is that most of the patients had cancer and developed the ventral hernia after an esophagectomy or gastrectomy for their disease. Many of these patients had received neoadjuvant chemotherapy or chemoradiotherapy shortly before resection and likely had impaired wound healing at their original operation. At the time of ventral hernia repair, most were still in active followup for their cancer and at risk for recurrence. Consequently, we had reservations about placement of a synthetic mesh which, if it became infected, could hamper chemotherapy or treatment of a recurrence. Most of our patients were getting routine CT scans as part of their cancer follow-up, which allowed us to observe the natural history of the XenMatrix onlay at 3-6 month intervals after placement. We observed that the mesh was still visible by $\mathrm{CT}$ in a patient at 18 months postoperatively, suggesting that the time to full integration of the XenMatrix mesh exceeds 12 months. Further support for this timeline comes from our re-operation 19 months after placing XenMatrix as a bridge for loss of domain in a patient. At reoperation, the mesh was still present, although it had separated into two layers with the superficial portion incorporated into the subcutaneous tissues and the deep portion adherent to the omentum and abdominal viscera. There was a recurrent hernia at the inferior portion of the mesh, but the mesh was still present over 1.5 years later. Importantly, there was no reconstructed abdominal wall when the mesh was used as a bridge, and similar to what other reports have shown, our findings confirm that if a biologic mesh is used as a bridge, then there will be a recurrent hernia [21]. In contrast, when the XenMatrix was used as an onlay over primary fascial closure, we found evidence for "proof of concept" of a biologic mesh in the patient that we re-explored for cancer at 4 months postimplant. Histologically, there was neovascularity going completely through the mesh into the subcutaneous tissue and evidence of fibroblast ingrowth into the mesh.

Our concept for ventral hernia repair with a biologic mesh is that the tension should be placed on the mesh and taken off of the primary midline fascial closure, rather than just laying the mesh over the midline closure as a buttress. The use of the lateral trans-fascial sutures allows significant tension to be placed on the mesh, and the onlay position of the mesh allows the midline to be closed and the amount of tension being placed on the mesh adjusted as the trans-fascial sutures are placed and tied. As the biologic mesh incorporates, tension is gradually transitioned to the native tissues. The importance of tension in wound healing has been studied by Culbertson et al. [29] who suggested that incisional abdominal hernias develop from early wound separation and failure, often within the first month, related to factors such as closure technique, wound ischemia, tension, or comorbid conditions that impair wound healing. However, they showed in a model of cultured fibroblasts that some tension is important to stimulate proliferation and orientation of fibroblasts and for collagen contraction [29]. This fits with our concept that gradual application of tension to the midline closure allows for initial secure healing and then subsequent strengthening as the native tissues assume more of the load during biologic mesh incorporation and replacement. Future studies and longer term follow-up will allow assessment of the role for and potential benefits of biologic XenMatrix mesh onlay for ventral hernia repair.

Acknowledgments This study was supported by a research grant from Davol Inc. (Warwick RI) for data collection.

Open Access This article is distributed under the terms of the Creative Commons Attribution Noncommercial License which permits any noncommercial use, distribution, and reproduction in any medium, provided the original author(s) and the source are credited.

\section{References}

1. Kingsnorth A, LeBlanc K (2003) Hernias: inguinal and incisional. Lancet 362:1561-1571

2. Choi JJ, Palaniappa NC, Dallas KB et al (2012) Use of mesh during ventral hernia repair in clean-contaminated and contaminated cases: outcomes of 33,832 cases. Ann Surg 255:176-180

3. Flum DR, Horvath K, Koepsell T (2003) Have outcomes of incisional hernia repair improved with time? A population-based analysis. Ann Surg 237:129-135 
4. Luijendijk RW, Hop WC, van den Tol MP et al (2000) A comparison of suture repair with mesh repair for incisional hernia. N Engl J Med 343:392-398

5. Ventral Hernia Working Group, Breuing K, Butler CE et al (2010) Incisional ventral hernias: review of the literature and recommendations regarding the grading and technique of repair. Surgery 148:544-558

6. Ramirez OM, Ruas E, Dellon AL (1990) "Components separation" method for closure of abdominal-wall defects: an anatomic and clinical study. Plast Reconstr Surg 86:519-526

7. Shankaran V, Weber DJ, Reed RL 2nd, Luchette FA (2011) A review of available prosthetics for ventral hernia repair. Ann Surg 253:16-26

8. Burger JWA, Luijendijk RW, Hop WCJ et al (2004) Long-term follow-up of a randomized controlled trial of suture versus mesh repair of incisional hernia. Ann Surg 240:578-583 discussion 583-575

9. Awad ZT, Puri V, LeBlanc K et al (2005) Mechanisms of ventral hernia recurrence after mesh repair and a new proposed classification. J Am Coll Surg 201:132-140

10. Iqbal CW, Pham TH, Joseph A et al (2007) Long-term outcome of 254 complex incisional hernia repairs using the modified Rives-Stoppa technique. World J Surg 31:2398-2404

11. Harth KC, Broome AM, Jacobs MR et al (2011) Bacterial clearance of biologic grafts used in hernia repair: an experimental study. Surg Endosc 25:2224-2229

12. Melman L, Jenkins ED, Hamilton NA et al (2011) Early biocompatibility of crosslinked and non-crosslinked biologic meshes in a porcine model of ventral hernia repair. Hernia 15:157-164

13. Helton WS, Fisichella PM, Berger R et al (2005) Short-term outcomes with small intestinal submucosa for ventral abdominal hernia. Arch Surg 140:549-560 (discussion 560-542)

14. Gupta A, Zahriya K, Mullens PL et al (2006) Ventral herniorrhaphy: experience with two different biosynthetic mesh materials, Surgisis and Alloderm. Hernia 10:419-425

15. Bluebond-Langner R, Keifa ES, Mithani S et al (2008) Recurrent abdominal laxity following interpositional human acellular dermal matrix. Ann Plast Surg 60:76-80

16. Maurice SM, Skeete DA (2009) Use of human acellular dermal matrix for abdominal wall reconstructions. Am J Surg 197:35-42

17. Ko JH, Salvay DM, Paul BC et al (2009) Soft polypropylene mesh, but not cadaveric dermis, significantly improves outcomes in midline hernia repairs using the components separation technique. Plast Reconstr Surg 124:836-847
18. Hiles M, Record Ritchie RD, Altizer AM (2009) Are biologic grafts effective for hernia repair?: a systematic review of the literature. Surg Innov 16:26-37

19. Pomahac B, Aflaki P (2010) Use of a non-cross-linked porcine dermal scaffold in abdominal wall reconstruction. Am J Surg 199:22-27

20. Diaz-Siso JR, Bueno E, Pomahac B (2012) Abdominal wall reconstruction using a non-cross-linked porcine dermal scaffold: a follow-up study. Hernia 17:37-44

21. Byrnes MC, Irwin E, Carlson D et al (2011) Repair of high-risk incisional hernias and traumatic abdominal wall defects with porcine mesh. Am Surg 77:144-150

22. Rosen MJ, Reynolds H, Champagne B, Delaney CP (2010) A novel approach for the simultaneous repair of large midline incisional and parastomal hernias with biologic mesh and retrorectus reconstruction. Am J Surg 199:416-421

23. Rosen MJ DG, Itani KM, Butler C, Vargo D, Smiell J, Rutan R (2012) Evaluation of surgical outcomes of retro-rectus versus intraperitoneal reinforcement with bio-prosthetic mesh in the repair of contaminated ventral hernias. Hernia; published on-line 14 March 2012

24. Patel KM NM, Gatti M, Bhanot P (2011) Indications and outcomes following complex abdominal reconstruction with component separation combined with porcine acellular dermal matrix reinforcement. Ann Plast Surg; e-published 2011

25. Parra MW, Rodas EB, Niravel AA (2011) Laparoscopic repair of potentially contaminated abdominal ventral hernias using a xenograft: a case series. Hernia 15:575-578

26. den Hartog D, Dur A, Tuinebreijer WE, Kreis RW (2011) Open surgical procedures for incisional hernias (review). The Cochrane Library, Wiley, pp 1-33

27. Kaafarani HMA, Hur K, Hirter A et al (2009) Seroma in ventral incisional herniorrhaphy: incidence, predictors and outcome. Am J Surg 198:639-644

28. Klima DA, Brintzenhoff RA, Tsirline VB et al (2011) Application of subcutaneous talc in hernia repair and wide subcutaneous dissection dramatically reduces seroma formation and postoperative wound complications. Am Surg 77:888-894

29. Culbertson EJ, Xing L, Wen Y, Franz MG (2011) Loss of mechanical strain impairs abdominal wall fibroblast proliferation, orientation, and collagen contraction function. Surgery 150: $410-417$ 